DOI: https://doi.org/10.24297/jap.v16i1.8402

\title{
Fine-Structure Constant from Sommerfeld To Feynman
}

\author{
Michael A. Sherbon \\ Case Western Reserve University Alumnus, United States \\ michael.sherbon@case.edu
}

\begin{abstract}
The fine-structure constant, which determines the strength of the electromagnetic interaction, is briefly reviewed beginning with its introduction by Arnold Sommerfeld and also includes the interest of Wolfgang Pauli, Paul Dirac, Richard Feynman and others. Sommerfeld was very much a Pythagorean and sometimes compared to Johannes Kepler. The archetypal Pythagorean triangle has long been known as a hiding place for the golden ratio. More recently, the quartic polynomial has also been found as a hiding place for the golden ratio. The Kepler triangle, with its golden ratio proportions, is also a Pythagorean triangle. Combining classical harmonic proportions derived from Kepler's triangle with quartic equations determine an approximate value for the finestructure constant that is the same as that found in our previous work with the golden ratio geometry of the hydrogen atom. These results make further progress toward an understanding of the golden ratio as the basis for the fine-structure constant.
\end{abstract}

Keywords: Fine-Structure Constant, Fundamental Constants, History Of Physics, Golden Ratio, Quartic Equation.

\section{Introduction}

Writing on the history of physics, Stephen Brush says that in 1916:

Sommerfeld generalized Bohr's model to include elliptical orbits in three dimensions. He treated the problem relativistically (using Einstein's formula for the increase of mass with velocity), .... According to historian Max Jammer, this success of Sommerfeld's fine-structure formula '... served also as an indirect confirmation of Einstein's relativistic formula for the velocity dependence of inertia mass.' [1].

From John S. Rigden, "The fine-structure constant derives its name from its origin. It first appeared in Sommerfeld's work to explain the fine details of the hydrogen spectrum. ... Since Sommerfeld expressed the energy states of the hydrogen atom in terms of the constant [alpha], it came to be called the fine-structure constant." [2] and more from Michael Eckert [3]. The fine-structure constant has also been called Sommerfeld's constant.

From Helge Kragh, "Sommerfeld's fine-structure theory was generally considered to be excellently and unambiguously confirmed by experiment. Because the theory rested on the foundation provided by Bohr, the experiments were also taken as strong support for his theory of atomic structure." [4]. Also, as Michael Eckert has noted, Sommerfeld has sometimes been compared to Kepler [5].

The electromagnetic coupling constant determining the strength of its interaction is the fine-structure constant

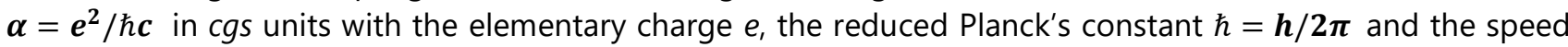
of light $c$. ." Arnold Sommerfeld states, "In our theory of the fine structure there is a confluence of the three main currents of modern research in theoretical physics, namely, the theory of electrons, the theory of quanta, and 
the theory of relativity" and the fine-structure constant "... could be interpreted more physically as the ratio of an electron's velocity in the first Bohr orbit to the speed of light." [6].

Helge Kragh writes:

Like many contemporary physicists, Dirac believed that ultimately $\alpha$ should be explainable by physical theory. As late as 1978, he wrote: 'The problem of explaining this number [fine-structure constant] is still completely unsolved. ... I think it is perhaps the most fundamental unsolved problem of physics at the present time, and I doubt very much whether any really big progress will be made in understanding the fundamentals of physics until it is solved.' [7].

This was a view also shared by Max Born, Werner Heisenberg and Wolfgang Pauli [8]. From Pauli, "The theoretical determination of the fine structure constant is certainly the most important of the unsolved problems of modern physics." [9]. Richard Feynman said it was the greatest mystery of physics, perhaps the most often quoted physicist on the fine-structure constant.

Describing Sommerfeld's work with Felix Klein, Pauli writes:

The standard treatise on the 'theory of the top,' which he wrote in conjunction with his teacher F. Klein in his early days, while he was still a 'Privatdozent' in Göttingen, and in which many technical problems are discussed, possesses a significance going far beyond applied mathematics. It contains, on the basis of work by Euler and Cayley, and of Hamilton's quaternions, the essential foundations of what considerably later was called the theory of representations of the rotation group in three-dimensional space. In particular, Klein had, following Cayley, clearly worked out the relation of this group to the 'covering group' of linear unitary unimodular transformations of two complex variables. Thus in this treatise, now a classic, the mathematical basis is developed for the two-component 'spinors' which turned up much later in wave mechanics [9].

In Wolfgang Pauli's summary of Sommerfeld's contributions to physics:

The intellectual tradition which Sommerfeld passed on to us will be transmitted to academic youth and thereby to posterity. This tradition goes back to Sommerfeld's teacher Felix Klein, and through him also to Riemann; indeed the grandly conceived work on the theory of the top, which Sommerfeld wrote with Klein, also contains the 'Cayley-Klein rotation parameters' which have become so important for the theory of spinors and hence also for Dirac's wave equations of the electron." [9]. Pauli also says, "Sommerfeld was versatile to an astonishing degree. He was a master of the technical applications of mathematics, of the partial differential equations of physics; of the formal classification of spectra; and again of wave mechanics, and in all alike he made decisive advances [9].

\section{Pythagorean mathematical history}

Willem Witteveen, writing on the Great Pyramid of Giza:

There is only one universal language, which is the language of numbers and proportions that are so striking and stunningly built into the Great Pyramid and to which our current science has no appropriate response. We can no longer ignore that this ancient civilization was aware of our units used in modern mathematics and physics and were even aware of our metric system. Our metric system originating in the eighteenth century, designed and implemented by a committee of mathematicians and physicists commissioned by the French revolutionary government." [10].

From the autobiography of Lyndon LaRouche, controversial and prolific publisher: 
... laws of astrophysics and microphysics, are also based on harmonic orderings congruent with the Golden Section. The 'fine structure constant,' which reflects the curvature of physical space time on both the astrophysical and microphysical scales, is one example of this. In a Gauss-Riemann mathematical physics, defined from the standpoint of a rigorous synthetic geometry, the constant speed of light and the quantum constant, also reflect, interdependently, the same connection. ... These constants are not properly mysterious; the example of Kepler's work already indicates their rational determination. ... The idea of Least Action in the universe, is a corollary of such rational determination of the necessity of constants [11].

Pierre Beaudry says, "... the Pythagorean method of spherical nesting of the regular solids, represented the actual 'missing link' between the ancient Egyptian knowledge of the pyramids and the Greeks, as well as the link between the astronomy of transoceanic-navigators, the Astronavigators, and the European legacy of science which was later established by Plato, Nicholas of Cusa, Kepler, Leibniz, Gauss, and Riemann." [12]. Beaudry also says this "... demonstrates that the principle of proportionality was the founding principle of scientific knowledge itself. ... You cannot square the circle, any more than you can cube the sphere. However, you can make them proportional." [12].

Pierre Beaudry continues, "For Pythagoras, the spherical composition of the five Platonic solids was the ultimate expression of the proportionality between the 'orbits of our reason' and the 'orbits of intelligence in the heavens.'" [12]. Also, "Thus, a single sphere of 16 great circles, entirely formed with Golden Sections, generates the five regular Platonic solids and creates the Great Pyramid Paradox from the higher power of the complex domain." [13]. Finally, "Great Pyramid of Egypt, and the Five Platonic Solids, are all historically bounded together and can never be separated from their common generative principle, which resides outside of them; and, the cement that bonds them together is the paradox of Squaring the Circle." [13].

\section{Bruce Director says:}

Plato cites the spinning top to show that it is physical motion that defines space and time, not, as the Eleatics maintained, a priori absolute space and time that defines motion.... At this point in our investigation the example of the simple spinning top becomes an insufficient example of a physical expression of still higher forms of hypergeometries. But if we follow the top's motion, as Riemann indicated, into the astrophysical and microphysical domains, such higher forms of hypergeometries emerge. For example, consider the actual motion of the Earth, around its axis, around the Sun and precession; or the motion within and among the galaxies; or the motions in the sub-atomic domains indicated by the experimental evidence of physical chemistry [14].

From Bruce Director again:

Once Kepler liberated science from Aristotle's chains of perfect circle to the more perfect freedom of eccentric or-bits, the question he confronted was, 'What was the principle that determined these eccentricities?' To answer this question he turned to the Pythagorean concept of harmonics. As he emphasized in his Harmonies of the World, the concept signified by the Greek word harmonia, or its Latin equivalent, congruencia, concerns the effect of unseen principles on the interaction among things in the sensible world.... Kepler utilized this method of harmonics to dis-cover the principle that governed the eccentric motions of the planets [15].

Arnold Sommerfeld from 1925, "Kepler should have experienced today's quantum theory. He would have seen the boldest dreams of his youth realized, not, admittedly, in the macrocosm of the stars, but in the microcosm of the atom. The shell structure of the atom is even more wonderful than the cosmography longed for by Kepler." [16]. 
Sommerfeld, as quoted by Wolfgang Pauli, "All integral laws of spectral lines and of atomic theory spring originally from the quantum theory. It is the mysterious organon on which Nature plays her music of the spectra, and according to the rhythm of which she regulates the structure of the atoms and nuclei." [9].

\section{Calculations of the fine-structure constant}

Helge Kragh states that, "By 1929 the fine-structure constant was far from new, but it was only with Eddington's work that the dimensionless combination of constants of nature was elevated from an empirical quantity appearing in spectroscopy to a truly fundamental constant." [7]. Sir Arthur Eddington believed the inverse of the fine-structure constant was the whole number 136, which he later amended to 137 . His work with advanced algebras was suggestive toward the following quartic equation from our previous work. [17]. This calculation of the inverse fine-structure constant gives the same approximate value as ancient geometry combined with the extension of Raji Heyrovska's work on the golden ratio structure of the hydrogen atom [18].

The inverse fine-structure constant is a root of:

$x^{4}-136 x^{3}-136 x^{2}-818 x+1=0$

This equation gives a value of for $x$ as $\alpha^{-1} \simeq 137.035999$ 168. The latest value reported by the Gabrielse group

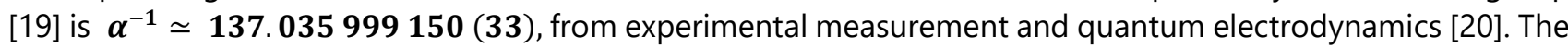
other root of the equation is approximately $1 / 818$ and $818=(4 \times 136)+(2 \times 137)$. Also of note is that the inflection points of the quartic are also related to the golden ratio [21]-[24]. Lin McMullin: "The golden ratio and its conjugate are lurking in every quartic polynomial." [24]. The golden ratio $\varphi=(\mathbf{1}+\sqrt{\mathbf{5}}) / 2$ and is a root of its minimal polynomial $x^{2}-x-1$. Both the golden ratio and its conjugate are roots of the quartic equation $x^{4}-$ $2 x^{3}-2 x+x+1=0$.

The inverse fine-structure constant $\boldsymbol{\alpha}^{\mathbf{- 1}}$ is a root of:

$$
x^{4}-137 x^{3}-10 x^{2}+697 x-365=0
$$

This equation gives the same approximate value for the inverse fine-structure constant as Eq. (1). $\mathbf{6 9 7}-\mathbf{1 3 7}=$ $\mathbf{2 8 0}+\mathbf{2 8 0} . \mathbf{3 6 5}+\mathbf{3 6 5}-\mathbf{1 0}=\mathbf{2 8 0}+\mathbf{4 4 0}=\mathbf{7 2 0}$. The scaling factor for the second pyramid on the Giza Plateau is 137 , having a height of $\mathbf{2} \times \mathbf{1 3 7}=\mathbf{2 7 4}$ Royal Cubits and a base of $\mathbf{3} \times \mathbf{1 3 7}=\mathbf{4 1 1}$ Royal Cubits. The Great Pyramid has a combined height and base of $\mathbf{2 8 0}+\mathbf{4 4 0}=\mathbf{7 2 0}$ Royal Cubits, 280/220 $=\mathbf{1 4} / \mathbf{1 1} \simeq \sqrt{\boldsymbol{\varphi}}$ and $\mathbf{2 2 0} / \mathbf{1 3 6} \simeq \boldsymbol{\varphi}$. Colonel R. S. Beard states that, "Sir William Petrie himself was thoroughly convinced that the Egyptians constructed the pyramid with a height-to-width-of-base ratio of seven to eleven." [25].

Another quartic equation with the Eq. (1) value with $x \simeq \alpha^{-\mathbf{1}}$ is also a root of:

$4 x^{4}-547 x^{3}-157 x^{2}+29 x+369=0$

$280=157+(369 / 3) \cdot 440=547-157+29+29-4-4$. Another quartic polynomial also gives the same approximate value for $\boldsymbol{\alpha}^{-1}$. The inverse fine-structure constant $\alpha^{-1} \simeq \sqrt{\boldsymbol{p} / \boldsymbol{x}}$, with $\boldsymbol{p}=\mathbf{2 5 , 9 2 0}$ (Egyptian value for precession, Plato's Great Year) and $x$ is a root of:

$x^{4}-139 x^{3}-96 x^{2}+386 x+12=0$

This quartic has a root $x \simeq \sqrt{5} / \varphi$ with the golden ratio. $280=1+1+139+139$ and $\mathbf{4 4 0}=\mathbf{3 8 6}+\mathbf{1 3 9}-\mathbf{9 6}+$ 12 - 1. From the precession of the equinoxes via the twelve star Egyptian sphere, which includes the origin of the Great Pyramid design and the geometry of the Platonic solids: $\mathbf{2 5 , 9 2 0}=\mathbf{3 6} \times \mathbf{7 2 0}$. Precession is also a factor in explaining the fine-structure constant [26]. 
The 'Key' of the Great Pyramid is 528 [27] and the inverse fine-structure constant $\boldsymbol{\alpha}^{\mathbf{- 1}} \simeq \mathbf{5 2 8} / \boldsymbol{x}$, where $x$ is a root of:

$x^{4}-44 x^{3}+86 x^{2}+281 x-53=0$

This equation also gives the same approximate value for the inverse fine-structure constant as Eq. (1). The root $x \simeq \varphi+\sqrt{5} .280=281-1.440=528-44-44=10+63+86+281.528 / 136 \simeq 2 \pi / \varphi$.

The inverse fine-structure constant $\boldsymbol{\alpha}^{\mathbf{- 1}} \simeq \mathbf{4 3 2} / \boldsymbol{x}$, where 432 is the main scaling factor for the Great Pyramid and $x$ is a root of:

$26 x^{4}-51 x^{3}-430 x^{2}+61 x+300=0$

This equation also gives the same approximate value for the inverse fine-structure constant as Eq. (1). $\boldsymbol{x} \simeq$ $4 / \sqrt{\varphi}$ and $528 / 432=11 / 9 \simeq 2 / \varphi \simeq 432 / 360=864 / 720$. Also, $25,920 / 432=60 . \quad \sqrt{432} \simeq 20.7 \simeq 1$ Royal Cubit and 440 Royal Cubits equal 432 long cubits of 21 inches. $432 \simeq \varphi \sinh (2 \pi)$, see the reference to Alfred Landé in our previous work [17]. $432 / \boldsymbol{\pi} \simeq 360 / \varphi^{2}$, the golden angle. From Witteveen, "The natural frequency of $432 \mathrm{Hertz}$ and the precession cycle of the Earth of 25,920 years together form the heartbeat of the Earth." [10].

Another approximation with the Eq. (1) value involves $\alpha^{-\mathbf{1}} \simeq \mathbf{8} \pi \boldsymbol{x}$, where $x$ is a root of:

$63 x^{4}-280 x^{3}-364 x^{2}+40 x+309=0$

$\mathbf{2 8 0} / \mathbf{4 0}=\mathbf{7}, 63 / 7=9,(9+9-7) \times \mathbf{4 0}=\mathbf{4 4 0}$. The root $x \simeq \varphi \varphi_{f}$, where $\varphi_{f}$ is the reciprocal Fibonacci constant. The $8 \pi$ is found in the base of William Eisen's Great Pyramid design along with the harmonic of the inverse of Newton's gravitational constant. [28].

Another approximation from our previous work [17] gives the same approximate value for the inverse finestructure constant. $\alpha^{-1} \simeq \varphi x$, where $x$ is a root of:

$3 x^{4}-250 x^{3}-346 x^{2}+48 x-36=0$

Along with $280=36-3-3+250, \quad 440=36-346+(3 \times 250)$ and $346=48+48+250$. Also, $440 \simeq$ $2 \varphi \times 136$, reference our previous extension of Eddington's work [17].

From the Pythagorean perspective the Foundation Stone number 729 is a harmonic of the fine-structure constant. Another equation that also gives the same approximate value for the inverse fine-structure constant as Eq. (1) is $\alpha^{-1} \simeq 729 / x$, where $x$ is a root of:

$10 x^{4}-37 x^{3}-113 x^{2}+73 x+371=0$

The root $x \simeq \varphi \varphi_{f}$, where $\varphi_{f}$ is the reciprocal Fibonacci constant, same root as Eq. (7) and the root of $\alpha^{-1} \simeq$ $\sqrt{p / x}$ of Eq. (4) is $x \simeq \sqrt{5} / \varphi \simeq 729 / 528 . \quad 280=10+10-37-73+(10 \times 37)$ and $440=73+371=$ $((113-73) / 10)$. Also, $\alpha^{-1} \simeq \mathbf{7 2 0} / x$ gives a quartic equation with the Eq. (1) value, a root $2 \varphi^{2}$ and coefficients related to Eq. (5).

Czech physicist Raji Heyrovska says, "On noticing the closeness of the fine structure constant ... to the ratio of the angles, $360^{\circ} / \varphi^{2} \ldots$ the author suggested that the small difference ... could be due to the Sommerfeld's relativity correction factor." [29]. "It was also pointed out that the ratio $\mathbf{3 6 0} / \boldsymbol{\varphi}^{\mathbf{2}} \ldots$ which is a Golden section of $360^{\circ}$, differs from the inverse fine structure constant by ... $2 / \varphi^{3} \ldots$ probably due to the difference in the $\mathrm{g}$ factors for the electron and proton ...," with the result of $\alpha^{-1} \simeq\left(360 / \varphi^{2}\right)-\left(2 / \varphi^{3}\right)$ [30]. 
$\alpha^{-1} \simeq \frac{360}{\varphi^{2}}-\frac{2}{\varphi^{3}}+\frac{A^{2}}{K \varphi^{4}}-\frac{A^{3}}{K^{2} \varphi^{5}}+\frac{A^{4}}{K^{3} \varphi^{7}}$

This extension of Heyrovska's equation also gives $\boldsymbol{\alpha}^{\mathbf{- 1}} \simeq \mathbf{1 3 7 . 0 3 5} 999$ 168. William Eisen describes the geometry of what he called the 'Golden Apex of the Great Pyramid' where dividing the sides of his mathematical model for the Great Pyramid by $\pi$ lengths along with four curves of the exponential function results in a small square in the center called the Golden Apex, the geometry and symmetry thought to be associated with the generation of the four fundamental forces of nature [28].

The Golden Apex $A$ is the side length of the resulting square. $\boldsymbol{A}=\boldsymbol{e}^{\boldsymbol{\pi}}-\mathbf{7} \boldsymbol{\pi}-\mathbf{1} \simeq \sqrt{\boldsymbol{\pi} \boldsymbol{\varphi}} \simeq \mathbf{3 / 2 0}$. The inverse Kepler-Bouwkamp constant is the polygon circumscribing constant $K \simeq \boldsymbol{\varphi}^{2} / \mathbf{2 A}$. Also of interest, $\ln (\boldsymbol{\varphi} / \boldsymbol{\alpha}) \simeq$ $\varphi / 2 A \simeq K / \varphi$ and $11 / 37 \simeq 2 A[28]$.

Raji Heyrovska found it a "... surprise to find for the first time that the Bohr radius is divided into two unique sections at the point of electrical neutrality, which is the Golden point. The Golden ratio, which manifests itself in many spontaneous creations of Nature, was thus found to originate right in the core of atoms." [30].

\section{Conclusion}

Pauli states in his article describing Sommerfeld's contributions, "I would not hesitate to set as superscription over Sommerfeld's works in a wider sense the title of Kepler's magnum opus--Harmonices mundi." [9]. Pauli continues, "I was well aware, as a pupil of Sommerfeld's, how these Pythagorean elements appearing in Kepler retain their vitality even today. ... That ancient spiritual 'dynamis' of number is still active, which was formerly expressed in the ancient doctrine of the Pythagoreans that numbers are the origin of all things and as harmonies represent unity in multiplicity." [9].

These calculations of the inverse fine-structure constant with the main parameters of the Great Pyramid have been directed toward a better understanding of the golden ratio as the basis for the fine-structure constant. In our previous work several more formulations for the fine-structure constant with the same approximate value have connections with prime number theory, the real fixed point of the hyperbolic cotangent, anomalous magnetic moment of the electron, Laplace limit of Kepler's equation and harmonic proportions of the Cosmological Circle [31, 32].

The proportion significant to 'squaring the circle' in the classical tradition was found by John Michell and presented in his study of what he named the Cosmological Circle. The regular heptagon and the golden ratio are both closely associated with the classical geometry of 'squaring the circle.' [33].

The long standing mystery of the fine-structure constant has also been associated with the image making faculty of consciousness, an aspect of the Egyptian Mysteries and alluded to in modern day accounts of initiation [34][37]. Wolfgang Pauli on the subject-object manifold:

The process of understanding in nature, together with the joy that man feels in understanding, i.e., becoming acquainted with new knowledge, seems therefore to rest upon a correspondence, a coming into congruence of pre-existent internal images of the human psyche with external objects and their behavior. This view of natural knowledge goes back, of course, to Plato and was ... also very plainly adopted by Kepler. ... [On Sommerfeld] It is as though there was here an echo of Kepler's search for the harmonies in 
the cosmos, guided by the musical feeling for the beauty of just proportion in the sense of Pythagorean philosophy, - an echo of his ... (geometry is the archetype of the beauty of the universe) [9].

\section{Conflicts of Interest}

No conflicted of interest is declared.

\section{Funding Statement}

No external funding provided.

\section{Acknowledgement}

Special thanks to Case Western Reserve University, MathWorld and WolframAlpha.

\section{References}

1. Brush, S.G. Making 20th Century Science: How Theories Became Knowledge, Oxford, UK: Oxford University Press, 2015, 220.

2. Rigden, J.S. Hydrogen: The Essential Element, Cambridge, MA: Harvard University Press, 2003, 55.

3. Eckert, M. "How Sommerfeld extended Bohr's Model of the Atom (1913-1916)," The European Physical Journal H, 39, 2, 141-156 (2014).

4. Kragh, H. Niels Bohr and the Quantum Atom: The Bohr Model of Atomic Structure 1913-1925, Oxford, UK: Oxford University Press, 2012, 162.

5. Eckert, M. Arnold Sommerfeld: Science, Life and Turbulent Times 1868-1951, New York, NY: Springer, 2013.

6. Sommerfeld, A. Atomic Structure and Spectral Lines, New York, NY: Dutton, 1934.

7. Kragh, H. "Magic Number: A Partial History of the Fine-Structure Constant," Archive for History of Exact Sciences, 57, 5, 395-431 (2003).

8. Sherbon, M.A. "Wolfgang Pauli and the Fine-Structure Constant," Journal of Science, 2, 3, 148-154 (2012).

9. Pauli, W., Enz, C.P. \& Meyenn, K. Writings on Physics and Philosophy, Berlin: Springer, 1994.

10. Witteveen, W. The Great Pyramid of Giza: A Modern View on Ancient Knowledge, Kempton, IL: Adventures Unlimited Press, 2016, 347.

11. LaRouche, L. The Power of Reason, Washington, DC: Executive Intelligence Review, 1987, 48.

12. Beaudry, P. "Pythagorean Spherics: The Missing Link Between Egypt and Greece," 21st Century Science and Technology, 17, 2, 48-67 (2004).

13. Beaudry, P. "The Twelve Star Egyptian Sphere that Generated the Great Pyramid and the Platonic Solids," (2009). lymcanada.org

14. Director, B. "Riemann for Anti-Dummies: The View from the Top," (2006). lymcanada.org/67

15. Director, B. "Riemann for Anti-Dummies: Hypergeometric Harmonics," (2006). lymcanada.org/64 
16. Seth, S. Crafting the Quantum: Arnold Sommerfeld and the Practice of Theory, 1890-1926, Cambridge, MA: The MIT Press, 2010, 201.

17. Sherbon, M.A. "Physical Mathematics and The Fine-Structure Constant," Journal of Advances in Physics, 14, 3, 5758-64 (2018).

18. Sherbon, M.A. "Fine-Structure Constant from Golden Ratio Geometry," International Journal of Mathematics and Physical Sciences Research, 5, 2, 89-100 (2018).

19. Gabrielse, G., Fayer, S.E., Myers, T.G. \& Fan, X. "Towards an Improved Test of the Standard Model's Most Precise Prediction," Atoms, 7, 2, 45 (2019).

20. Aoyama, T., Kinoshita, T., \& Nio, M. "Theory of the Anomalous Magnetic Moment of the Electron," Atoms, 7 , 1, 28 (2019).

21. Aude, H.T.R. "Notes on Quartic Curves," The American Mathematical Monthly, 56, 3, 165-170 (1949).

22. Rinvold, R.A. "Fourth Degree Polynomials and the Golden Ratio," The Mathematical Gazette, 93, 527, 292295 (2009).

23. Totland, H. "Quartic Polynomials and the Golden Ratio," Mathematics Magazine, 82, 3, 197-201 (2009).

24. McMullin, L. "The Golden Ratio and Quartic Polynomials," (2016). teachingcalculus.files.wordpress.com/2016

25. Beard, R.S. "The Fibonacci Drawing Board Design of the Great Pyramid of Gizeh," The Fibonacci Quarterly, 6, 85-87 (1968).

26. Griffiths, D.J. \& Schroeter, D.F. Introduction to Quantum Mechanics, Cambridge, UK: Cambridge University Press, 2018.

27. Sherbon, M.A. "Quintessential Nature of the Fine-Structure Constant," Global Journal of Science Frontier Research, $A, 15,4,23-26$ (2015).

28. Eisen, W. "The Mysteries of the Constants (e) and (i)," in The Universal Language of Cabalah, Marina Del Rey, CA: DeVorss, 1989, 160-178.

29. Heyrovska, R. "The Golden Ratio, lonic and Atomic Radii and Bond Lengths," Molecular Physics, 103, 877 882 (2005).

30. Heyrovska, R. "Golden Ratio Based Fine Structure Constant and Rydberg Constant for Hydrogen Spectra," International Journal of Sciences, 2, 5, 28-31 (2013).

31. Sherbon, M.A. "Fundamental Nature of the Fine-Structure Constant," International Journal of Physical Research, 2, 1, 1-9 (2014).

32. Sherbon, M.A. "Fundamental Physics and the Fine-Structure Constant," International Journal of Physical Research, 5, 2, 46-48 (2017).

33. Michell, J. "Is the Fibonacci Sequence or Golden Ratio Represented in the Dimensions of the Great Pyramid of Egypt?" The Fibonacci Newsletter (1974).

34. Eisen, W. The English Cabalah Volume II: The Mysteries of Phi, Marina Del Rey, CA: DeVorss, 1982.

35. Lamy, L. Egyptian Mysteries: New Light on Ancient Knowledge, New York, NY: Thames \& Hudson, 1997. 
36. Chaney, E. Initiation in the Great Pyramid, Upland, CA: Astara, 1987.

37. Ellis, N. Imagining the World into Existence: An Ancient Egyptian Manual of Consciousness, Rochester, VT: Bear \& Company, 2012. 